\title{
La malattia di Fabry: una sindrome cardio-renale da malattia sistemica
}

\author{
Fulvio Floccari ${ }^{1}$, Rodolfo Rivera ${ }^{2}$, Moreno Malaguti ${ }^{1}$, Alberto Santoboni ${ }^{3}$, \\ Vincenzo Barbera ${ }^{3}$, Luca Di Lullo ${ }^{3}$
}

\author{
${ }^{1}$ Centro di Riferimento Regionale per la Nefrologia e la Dialisi "M. Biagini”, Civitavecchia (Roma) \\ ${ }^{2}$ UOC Nefrologia e Dialisi, Ospedale San Gerardo, Monza \\ ${ }^{3}$ UOC Nefrologia e Dialisi, Ospedale Parodi Delfino, Colleferro (Roma)
}

FABRY'S DISEASE: A TYPE 5 CARDIORENAL SYNDROME

Abstract. Fabry disease is an inherited lysosomal storage disorder in which chronic accumulation of globotriaosylceramide (Gb3) induces renal, cardiac and neurological damage. Linked to chromosome $X$, it has an estimated incidence of one case every 55,000 male live births, up to one every 3,100 .

Enzyme replacement therapy is proposed as treatment of choice for this condition and is able to modify substantially the clinical history.

This review is intended as a stimulus to look with the eyes of a "cardionephrologist" at this systemic cardiorenal syndrome.

Key words: Fabry disease, End stage renal disease, Cardiomyopathy, Arrhythmias, Pain

Conflict of interest: None.

Ricevuto: 31 Gennaio 2013; Accettato: 19 Febbraio 2013

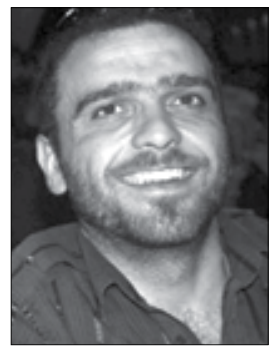

\section{Introduzione}

La malattia di Fabry è una disfunzione lisosomiale ereditaria, nella quale l'accumulo cronico di globotriaosilceramide (Gb3) induce danno d'organo renale, cardiaco e neurologico. Come vedremo nel corso di questa rassegna, la presenza di danno renale modifica la prognosi e influenza pesantemente la risposta alla F.ulvio Floccari terapia, ponendo la malattia di Fabry tra le sindromi cardio-renali di tipo 5 , secondo la classificazione dell'Acute

Dialysis Quality Initiative Group (1).

Trasmessa attraverso il cromosoma $\mathrm{X}$ e con penetranza di tipo recessivo, la causa molecolare della malattia consiste in una mutazione del gene GLA, che codifica l'enzima lisosomiale alfa galattosidasi A ( $\alpha$-GAL A) e nel conseguente progressivo accumulo di $\mathrm{Gb} 3$ nei lisosomi. Tale accumulo conduce a una disfunzione cellulare prevalentemente endoteliale, che comporta un insulto ischemico e infiammatorio, prevalentemente a carico di rene, miocardio, tessuto connettivo e sistema nervoso (2).

L'angiocheratoma corporis diffusum è stato descritto per la prima volta da Johann Fabry in Germania e da William Anderson nel Regno Unito nel 1898 attraverso due diverse riviste scientifiche $(3,4)$. Il difetto enzimatico responsabile della malattia è stato però identificato solo nel 1967 (5).

\section{Epidemiologia}

L'incidenza della malattia di Fabry è stimata in un range molto ampio, che va da un caso ogni 55000 maschi nati vivi sino a uno ogni 3100 (6). Tale grande variabilità è legata alla presenza di tante varianti fenotipiche, molte delle quali caratterizzate da manifestazioni sub-cliniche o a insorgenza tardiva (6) e da conseguenti ritardi o fallimenti nella diagnosi.

\section{Le possibili varianti}

I maschi, caratterizzati da un unico cromosoma $\mathrm{X}$, presentano esclusivamente il gene mutante e, quindi, hanno come caratteristica l'assenza pressoché totale di $\alpha$-GAL A. I sintomi precoci (parestesie dolorose alle estremità, febbre, ipoidrosi, opacità corneali e angiocheratomi) insorgono solitamente nella II-III decade di vita, pur essendone stati riportati casi di insorgenza anche in età pediatrica.

Il progressivo coinvolgimento cronico di rene, cuore e sistema nervoso centrale rappresenta la causa delle elevate morbilità e mortalità della malattia di Fabry nel genere maschile (2).

Il coinvolgimento renale è spesso annunciato dalla proteinuria, che, talvolta, insorge già in età adolescenziale, e mostra la tendenza a progredire sino all'uremia terminale nella quarta decade di vita $(2,7)$.

L'accumulo di Gb3 a livello cardiaco riguarda il tessuto di conduzione, i miociti e le valvole cardiache, con la conse- 
guenza di produrre aritmie, valvulopatie e ipertrofia ventricolare sinistra, scompenso cardiaco congestizio e cardiopatia ischemica (8-10).

L'accumulo di sfingolipidi a livello cerebrale ha invece come conseguenza lo sviluppo di condizioni di ischemia più o meno silenti, tanto da condurre ad attacchi ischemici transitori e stroke (11).

In una minoranza di pazienti maschi è ancora presente una seppur minima attività enzimatica della $\alpha$-GAL A, corrispondente a circa il $10 \%$ delle normali concentrazioni sieriche. Tipicamente tali pazienti manifestano unicamente il coinvolgimento cardiaco della malattia, per cui ci si riferisce a tale condizione come alla "variante cardiaca" della malattia di Fabry (12, 13).

Per analogia, alcuni pazienti di genere maschile, caratterizzati da una attività di $\alpha$-GAL A residua ulteriormente depressa $(<5 \%)$ e affetti esclusivamente dalle manifestazioni renali della malattia, suggeriscono l'esistenza di una "variante renale" della stessa (14-16).

Le donne, ovviamente caratterizzate dalla presenza di due cromosomi X e dal processo della lyonizzazione di uno dei due, mostrano un quadro genomico più complesso e misterioso, pur essendo pressoché invariabilmente eterozigoti per la mutazione. Come conseguenza di tali meccanismi, le pazienti del gentil sesso possono presentare un'attività enzimatica sierica di $\alpha$-GAL $A$ in un range che spazia dalla assoluta normalità a valori estremamente bassi, senza che esistano correlazioni certe tra i livelli sierici di attività enzimatica e la severità delle manifestazioni cliniche. In tali pazienti lo spettro della malattia può variare grandemente dalla assoluta asintomaticità al severo coinvolgimento multi-organo (2), riportato comunque in un terzo soltanto di queste pazienti (17).

\section{Il coinvolgimento cardiaco}

La sintomatologia cardiaca caratteristica dei pazienti affetti da malattia di Fabry comprende la dispnea, le palpitazioni, la sincope e l'angina.

Le aritmie sono presenti nel $60 \%$ circa dei pazienti affetti dalla forma classica della malattia, mentre più rare sono le anomalie valvolari e la cardiomiopatia. La variante cardiaca si manifesta invece quasi esclusivamente attraverso l'ipertrofia ventricolare sinistra, a presentazione tardiva (18).

La causa fisiopatologica di tali aritmie risiederebbe proprio nell'accumulo di glicosfingolipidi, talmente massivo da giungere a rappresentare sino al 3\% della massa miocardica totale, fino ad arrivare alla conseguente fibrosi miocardica (19).

Le aritmie atriali sono le più comuni e, in particolare, è frequente la fibrillazione atriale, che ha un'incidenza quattro volte superiore rispetto a quella della popolazione generale. Forti predittori di rischio di sviluppare tale aritmia sarebbero l'età, la dilatazione atriale sinistra, la massa ventricolare sinistra e la presenza di episodi di angina (20).

La tachicardia ventricolare non sostenuta si è rivelata anch'essa un'aritmia relativamente frequente ( $8 \%$ in una casistica di 78 pazienti), strettamente correlata alla presenza di ipertrofia ventricolare sinistra (21).

Sono poi state descritte varie tipologie di blocchi di conduzio- ne, tali da richiedere l'impianto di pacemaker nel 10\% circa dei pazienti (21).

Il tratto elettrocardiografico più comune è il riscontro delle stigmate dell'ipertrofia ventricolare sinistra (20). Tale aspetto è di grande utilità nella diagnosi differenziale elettrocardiografica con l'amiloidosi, nel corso della quale è, al contrario frequente, osservare la presenza di bassi voltaggi.

La cardiomiopatia della malattia di Fabry è essenzialmente un'ipertrofia ventricolare sinistra con note di cardiomiopatia restrittiva (20). I cardiomiociti sono più grandi del normale e presentano, all'analisi ultrastrutturale, vacuoli peri-nucleari ripieni di Gb3 (20). La fibrosi interstiziale è diffusa e ingravescente nel tempo. Le arteriole intramiocardiche sono ispessite a causa dell'ipertrofia e della proliferazione delle cellule muscolari lisce e dell'infarcimento dell'endotelio e delle stesse cellule muscolari da parte degli sfingolipidi (22).

Il pattern geometrico più diffuso è quello da ipertrofia concentrica ( $>50 \%$ dei soggetti di sesso maschile), mentre è più raro osservare forme di ipertrofia a prevalenza settale (23-28). Al contrario delle comuni forme di ipertrofia ventricolare sinistra non fisiologica, la causa ultra-strutturale dell'ipertrofia non è, però, qui, l'accumulo di proteine contrattili anomale ma, ovviamente, di sfingolipidi. Come conseguenza di tale diversità, la disfunzione diastolica della malattia di Fabry è solitamente solo lieve-moderata (quindi meno grave di quella tipica delle altre tesaurismosi) e la funzione sistolica è solitamente preservata (23-28).

Le possibili conseguenze del rimodellamento dei vasi intramiocardici sul rischio coronarico rappresentano un dato controverso. Le evidenze di letteratura sembrano escludere infatti una maggiore incidenza di infarti del miocardio o di procedure di rivascolarizzazione $(29,30)$. D'altra parte, è stata riportata una maggiore incidenza di episodi di angina, particolarmente tra i pazienti affetti da ipertrofia ventricolare sinistra $(29,30)$. Le anomalie valvolari sono riportate in una percentuale prossima al 50\% dei pazienti affetti, anche se raramente con gradi elevati di severità. La forma più comune di anomalia valvolare è l'insufficienza mitralica, di grado moderato. A conferma della benignità dell'interessamento valvolare vi è la ridotta richiesta di chirurgia correttiva $(20,31)$ in tali pazienti.

\section{Il coinvolgimento renale}

Il danno renale è causato dalla diffusa deposizione di GL3 nei glomeruli, nel sistema tubulare e nell'albero vascolare renale. Alla microscopia ottica l'aspetto dei glomeruli è caratteristicamente "schiumoso", con rigonfiamento diffuso e vacuolizzazione dei podociti. La microscopia elettronica mostra podociti e cellule mesangiali ricche di granuli lisosomiali elettron-densi, arrangiati con un pattern lamellare. La patogenesi della nefropatia di Fabry non è stata comunque ancora definita in maniera univoca.

Un sistema di score per le biopsie renali è stato proposto dal Gruppo di Studio Internazionale della Nefropatia di Fabry (32), come tentativo di standardizzare la lettura delle biopsie renali e di sviluppare un indice di cronicità del danno, a scopo di indagine prognostica e di previsione della risposta alla terapia enzimatica. 
La microalbuminuria e la proteinuria sono le manifestazioni più precoci della nefropatia, identificabili con i comuni test diagnostici spesso già in età giovanile. Tipicamente, una riduzione del GFR insorge invece nella terza decade di età dei maschi affetti. In una casistica del National Institute of Health (NIH) di Bethesda, in un periodo di osservazione di oltre 25 anni, il $50 \%$ dei pazienti mostrava segni di nefropatia all'età di 42 anni, mentre ciò era vero nel 100\% della coorte oltre i 55 anni (33). La prevalenza della nefropatia di Fabry tra tutte le cause di uremia terminale è riportata tra lo $0.017 \%$ degli Stati Uniti d'America e lo $0.019 \%$ dell'Europa, con una morbilità e una mortalità più elevate rispetto alla popolazione generale in dialisi $(34,35)$.

Numerosi studi di screening riportano peraltro un numero molto elevato di casi non diagnosticati, il che correggerebbe il dato di prevalenza allo $0.16 \%$ dell'Austria o addirittura all'1.2\% del Giappone $(36,37)$.

\section{Il coinvolgimento neurologico}

Le disfunzioni neurologiche più diffuse sono le vertigini, la diplopia, l'emiatassia e le complicazioni ischemiche. La presenza di diffuse lesioni della materia bianca profonda è frequentemente rilevata alla risonanza magnetica anche in soggetti giovani (38). La disfunzione endoteliale si è dimostrata la causa più rilevante delle turbe del flusso ematico cerebrale, della reattività vascolare endotelio-mediata e del metabolismo neuronale (39-41). Studi di screening riportano la prevalenza di stroke a eziologia sospetta nel $4.9 \%$ dei maschi affetti e nel $2.4 \%$ delle femmine (41).

\section{La terapia}

Storicamente, il trattamento della malattia di Fabry è stato non specifico, sintomatico e palliativo, basato sull'utilizzo di anti-ipertensivi, antiaggreganti, gabapentin e carbamazepina. Il primo trial con $\alpha$-GAL A risale agli anni Ottanta, ma l'impossibilità di produrre grandi quantitativi di enzima con tecniche industriali ha impedito l'applicazione clinica dei risultati di questo trial $(42,43)$.

Solo recentemente tale problematica è stata risolta con la produzione di $\alpha$-GAL A ricombinante umana, approvata dall'EMEA nel 2001 e dalla FDA nel 2003 per il trattamento della malattia di Fabry.

Già trial preclinici hanno dimostrato sicurezza ed efficacia della terapia enzimatica $(44,45)$. Uno studio di fase 3 con agalsidase beta in 58 pazienti ha dimostrato una pressoché completa clearance dei depositi di GL3 a livello endoteliale renale, cardiaco e cutaneo (46). Pazienti arruolati in trial open-label hanno confermato sicurezza ed efficacia del trattamento protratto per oltre quattro anni (47).

Ancora un altro RCT ha dimostrato un significativo miglioramento della sintomatologia collegata alla neuropatia (48).

Tutti questi trial promettono che la terapia enzimatica sia di grande efficacia nella prevenzione e nella riduzione della progressione del danno d'organo, se applicata in pazienti ancora giovani e nei primi stadi di evoluzione della malattia, particolarmente quando non è ancora insorta la disfunzione renale. I risultati di trial open-label condotti in condizioni di maggiore progressione della malattia suggerirebbero outcome meno ottimistici. La disfunzione renale in particolare insieme alla proteinuria, quando già presenti all'atto dell'inizio della terapia enzimatica, sembrerebbero avere un significato prognostico negativo di grande rilievo (49) nell'efficacia della terapia.

Tale dato sembra non essere confermato dal Fabry Disease Clinical Trial Study Group (50), che riporta una riduzione dell'outcome combinato "primo evento" renale/cardiaco/cerebrovascolare o morte in una coorte di pazienti già nefropatici all'atto dell'ingresso nel trial. Aggiustando i risultati per la proteinuria all'ingresso nello studio, lo studio riporta una riduzione del rischio del 53\% $(\mathrm{p}=0.06)$ nella popolazione intention-to-treat e del $61 \%(\mathrm{p}=0.034)$ nella popolazione "per protocol".

Un'analisi secondaria di questo studio conferma però come l'efficacia del trattamento sia maggiore tra i pazienti con funzione renale maggiormente preservata (GFR $>55 \mathrm{~mL} / \mathrm{min} / 1.73$ $\mathrm{mq})$ e senza proteinuria.

Conseguentemente, qualunque trattamento di supporto capace di ridurre la proteinuria (attraverso l'inibizione del sistema renina angiotensina) è da ritenersi presumibilmente utile nella gestione della malattia di Fabry, pur in assenza di evidenze scientifiche in merito (51). Altrettanto è probabilmente da intendersi per le terapie di supporto, capaci di ridurre l'incidenza di eventi ischemici (attraverso gli antiaggreganti) e di correggere la dislipidemia (mediante l'utilizzo di statine).

La presenza di attività endogena di produzione di $\alpha$-Gal A nell'organo del donatore rende il trapianto di rene una soluzione efficace alla nefropatia di Fabry (52), capace di correggere $\mathrm{i}$ livelli sierici dell'enzima.

\section{Conclusioni}

L'introduzione della terapia enzimatica ricombinante ha prodotto progressi notevoli nella possibilità di trattare la malattia di Fabry e di prevenirne le complicanze. I dati in letteratura suggeriscono che la tempestività della diagnosi e dell'inizio della terapia sia cruciale per l'efficacia di quest'ultima.

La proteinuria è stata identificata come maggiore fattore di progressione della nefropatia e studi in corso stanno indagando circa l'utilità di un utilizzo combinato della terapia enzimatica e della terapia antiproteinurica massiva.

Guardare al paziente nefropatico con occhi da "cardionefrologi" ci consentirà di formulare più di frequente l'ipotesi diagnostica di malattia di Fabry, di intervenire con maggiore tempestività nella storia clinica di questa malattia e di segnare un altro punto nel match della prevenzione dell'insufficienza renale severa.

\section{Riassunto}

La malattia di Fabry è una disfunzione lisosomiale ereditaria, nella quale l'accumulo cronico di globotriaosilceramide (Gb3) induce danno d'organo renale, cardiaco e neurologico. Trasmessa attraverso il cromosoma $\mathrm{X}$, ha un'incidenza stimata che va da un caso ogni 55000 maschi nati vivi sino a uno ogni 3100.

La terapia enzimatica sostitutiva si propone come trattamento di elezione di tale patologia, capace di modificarne la sto- 
ria clinica in maniera sostanziale.

Questa review si propone come uno stimolo a guardare con occhi da "cardionefrologi" a questa sindrome cardio-renale sistemica.

Parole chiave: Malattia di Fabry, Sindrome cardiorenale, Rene, Cuore

Dichiarazione di conflitto di interessi: Gli Autori dichiarano di non avere conflitto di interessi.
Indirizzo degli Autori:

Dr. Fulvio Floccari

Centro Regionale di Riferimento Regionale per la Nefrologia

e la Dialisi "M. Biagini"

Ospedale San Paolo

Largo Donatori di Sangue 1

00053 Civitavecchia (Roma)

fulviofloccari@gmail.com

\section{Bibliografia}

1. Desnick RJ, Ioannou, Eng CM. Alpha-Galactosidase A deficiency: Fabry's disease. In Scriver CR, Beaudet AL, Sly WS, Valle D, Kinzler KE, Vogelstein B Eds. The metabolic and molecular bases of inherited disease. New York: McGraw-Hill 2001; 3733-74.

2. Anderson W. A case of 'angeiokeratoma'. Br J Dermatol 1898; 10: 113-7.

3. Fabry J. Ein Beitrag zur Kenntnis der Purpura haemorrhagica nodularis (Purpura papulosa haemorrhagica Hebrae). Arch Dermatol Syph 1898; 43: 187-200.

4. Brady RO, Gal AE, Bradley RM, Martensson E, Warshaw AL, Laster L. Enzymatic defect in Fabry's disease. Ceramidetrihexosidase deficiency. N Engl J Med 1967; 276: 1163-7.

5. Spada M, Pagliardini S, Yasuda M, et al. High incidence of later-onset Fabry disease revealed by newborn screening. Am J Hum Genet 2006; 79: 31-40.

6. Branton M, Schiffmann R, Kopp JB. Natural history and treatment of renal involvement in Fabry's disease. J Am Soc Nephrol 2002; 13 (Suppl. 2): S139-43.

7. Kampmann C, Baehner F, Ries M, Beck M. Cardiac involvement in Anderson-Fabry's disease. J Am Soc Nephrol 2002; 13 (Suppl. 2): S147-9.

8. Linhart A, Palcek T, Bultas J, et al. New insights in cardiac structural changes in patients with Fabry's disease. Am Heart J 2000; 139: 1101-8.

9. Goldman ME, Cantor R, Schwartz MF, Backer M, Desnick RJ. Echocardiographic abnormalities and disease severity in Fabry's disease. J Am Coll Cardiol 1986; 7: 1157-61.

10. Kolodny EH, Pastores GM. Anderson-Fabry's disease: extrarenal, neurologic manifestations. J Am Soc Nephrol 2002; 13 (Suppl. 2): S150-3.

11. Elleder M, Bradova V, Smid F, et al. Cardiocyte storage and hypertrophy as a sole manifestation of Fabry's disease. Report on a case simulating hypertrophic non-obstructive cardiomyopathy. Virchows Arch A Pathol Anat Histopathol 1990; 417: 449-55.

12. von Scheidt W, Eng CM, Fitzmaurice TF, et al. An atypical variant of Fabry's disease with manifestations confined to the myocardium. New Engl J Med 1991; 324: 395-9.

13. Meroni M, Spisni C, Tazzari S, et al. Isolated glomerular proteinuria as the only clinical manifestation of Fabry's disease in an adult male. Nephrol Dial Transplant 1997; 12: 221-23.

14. Desnick RJ, Wasserstein MP. Fabry's disease: clinical features and recent advances in enzyme replacement therapy. Adv Nephrol Necker Hosp 2001; 31: 317-39.

15. Nakao S, Komada C, Takenata T, et al. Fabry's disease: Detection of undiagnosed hemodialysis patients and identification of a "renal variant" phenotype. Kidney Int 2003; 64: 801-7.

16. MacDermot KD, Holmes A, Miners AH. Anderson-Fabry's disease: Clinical manifestations and impact of disease in a cohort of 60 obligate carrier females. J Med Genet 2001; 38: 769-75.

17. Mehta A, Ricci R, Widmer U, et al. Fabry disease defined: baseline clinical manifestations of 366 patients in the Fabry Outcome Survey. Eur J Clin Invest 2004; 34: 236-42.

18. Kampmann C, Linhart A, Baehner F, et al. Onset and progression of the Anderson-Fabry disease related cardiomyopathy. Int J Cardiol 2008; 130: 367-73.

19. Linhart A, Kampmann C, Zamorano JL, et al. Cardiac manifestations of Anderson-Fabry disease: results from the international Fabry out come survey. Eur Heart J 2007; 28: 1228-35.

20. Shah JS, Hughes DA, Sachdev B, et al. Prevalence and clinical significante of cardiac arrhythmia in Anderson-Fabry disease. Am J Cardiol 2005; 96: 842-6.

21. Chimenti C, Padua L, Pazzaglia C, et al. Cardiac and skeletal myopathy in Fabry disease: a clinicopathologic correlative study. Hum Pathol 2012; 43 (9): 1444-52.

22. Hughes SE, McKenna WJ. New insights into the pathology of inherited cardiomyopathy. Heart 2005; 91: 257-64.

23. Stöllberger C, Finsterer J. Extracardiac medical and neuromuscular implications in restrictive cardiomyopathy. Clin Cardiol 2007; 30: 375-80.

24. Wood MJ, Picard MH. Utility of echocardiography in the evaluation of individuals with cardiomyopathy. Heart 2004; 90 : 707-712.

25. Linhart A, Palecek T, Bultas J, et al. New insights in cardiac structural changes in patients with Fabry's disease. Am Heart J 2000; 139: 1101-8.

26. Sadick N, Thomas L. Cardiovascular manifestations in Fabry disease: a clinical and echocardiographic study. Heart Lung Circ 2007; 16: 200-6.

27. Shah JS, Lee P, Hughes D, et al. The natural history of left ventricular systolic function in Anderson-Fabry disease. Heart 2005; 91: 533-4.

28. Desnick RJ, Brady R, Barranger J, et al. Fabry disease, an under- 
recognized multisystemic disorder: expert recommendations for diagnosis, management, and enzyme replacement therapy. Ann Intern Med 2003; 138: 338-46.

29. Kampmann C, Baehner F, Whybra C, et al. Cardiac manifestations of Anderson-Fabry disease in heterozygous females. J Am Coll Cardiol 2002; 40: 1668-74.

30. Weidemann F, Strotmann JM, Niemann M, et al. Heart valve involvement in Fabry cardiomyopathy. Ultrasound Med Biol 2009; 35 (5): 730-5.

31. Fogo A, Oliveira JP, Waldeck S, Warnock DG. Developing a scoring system and chronicity index for kidney involvement in Fabry disease: report of the International Fabry Kidney Biopsy Working Group. J Am Soc Nephrol. 2006; 17: SAPO231.

32. Thadhani R, Wolf M, West ML, et al. Patients with Fabry disease on dialysis in the United States. Kidney Int 2002; 61: 249-55.

33. Tsakiris D, Simpson HK, Jones EH, et al. Report on management of renale failure in Europe, XXVI, 1995. Rare diseases in renal replacement therapy in the ERA-EDTA Registry. Nephrol Dial Transplant 1996; 11 (Suppl. 7): 4-20.

34. Kotanko P, Kramar R, Devrnja D, et al. Results of a nationwide screening for Anderson-Fabry disease among dialisi patients. J Am Soc Nephrol 2004; 15: 1323-9.

35. Nakao S, Kodama C, Takenaka T, et al. Fabry disease: detection of undiagnosed hemodialysis patients and identification of a "renal variant" phenotype. Kidney Int 2003; 64: 801-7.

36. Branton MH, Schiffmann R, Sabnis SG, et al. Natural history of Fabry renal disease: influence of alpha-galactosidase A activity and genetic mutations on clinical course. Medicine 2002; 81: 122-38.

37. Jardim L, Vedolin L, Schwartz IV, et al. CNS involvement in Fabry disease: clinical and imaging studies before and after 12 months of enzyme replacement therapy. J Inherit Metab Dis 2004; 27: 229-40.

38. Moore DF, Scott LT, Gladwin MT, et al. Regional cerebral hyperperfusion and nitric oxide pathway dysregulation in $\mathrm{Fa}$ bry disease: reversal by enzyme replacement therapy. Circulation 2001; 104: 1506-12.

39. Altarescu G, Moore DF, Pursley R, et al. Enhanced endothelium-dependent vasodilation in Fabry disease. Stroke 2001; 32: 1559-62.

40. Itoh Y, Esaki T, Cook M, et al. Local and global cerebral blood flow and glucose utilization in the alpha-galactosidase A knockout mouse model of Fabry disease. J Neurochem 2001;
79: 1217-24.

41. Rolfs A, Böttcher T, Zschiesche M, et al. Prevalence ofFabry disease in patients with cryptogenic stroke: a prospective study. Lancet 2005; 366: 1794-6.

42. Brady RO, Tallman JF, Johnson WG, et al. Replacement therapy for inherited enzyme deficiency. Use of purified ceramidetrihexosidase in Fabry's disease. N Engl J Med 1973; 289 : 9-14.

43. Desnick RJ, Dean KJ, Grabowski G, Bishop DF, Sweeley CC. Enzyme therapy in Fabry's disease: Differential plasma clearance and metabolic effectiveness of plasma and splenic alphagalactosidase A isozymes. Proc Natl Acad Sci USA 1979; 76 : 5326-30.

44. Eng CM, Banikazemi M, Gordon RE, et al. A phase 1/2 clinical trial of enzyme replacement in Fabry disease: pharmacokinetic, substrate clearance, and safety studies. Am J Hum Genet 2001; 68: 711-22.

45. Schiffmann R, Murray GJ, Treco D, et al. Infusion of alphagalactosidase A reduces tissue globotriaosylceramide storage in patients with Fabry disease. Proc Natl Acad Sci USA 2000; 97: 365-70.

46. Eng CM, Guffon N, Wilcox WR, et al. Safety and efficacy of recombinant human alpha-galactosidase A replacement therapy in Fabry's disease. N Engl J Med 2001; 345: 9-16.

47. Germain DP, Waldek S, Banikazemi M, et al. Sustained, longterm renal stabilization after 54 months of agalsidase beta therapy in patients with Fabry disease. J Am Soc Nephrol 2007; 18: $1547-57$.

48. Schiffmann R, Kopp JB, Austin HA 3rd, et al. Enzyme replacement therapy in Fabry disease: a randomized controller trial. JAMA 2001; 285: 2743-9.

49. Breunig F, Weidemann F, Strotmann J, Knoll A, Wanner C. Clinical benefit of enzyme replacement therapy in Fabry disease. Kidney Int 2006; 69: 1216-21.

50. Banikazemi M, Bultas J, Waldek S, et al. Agalsidase-beta therapy for advanced Fabry disease: a randomized trial. Ann Intern Med 2007; 146: 77-86.

51. Breunig F, Wanner C. Update on Fabry disease: kidney involvement, renal progression and enzyme replacement therapy. $\mathrm{J}$ Nephrol 2008; 21: 32-7.

52. Ojo A, Meier-Kriesche HU, Friedman G, et al. Excellent out come of renal transplantation in patients with Fabry's disease. Transplantation 2000; 69: 2337-9. 